\section{Ökologische Lern- und Entwicklungsprozesse von Unternehmen}

In der Literatur zum ökologisch bewußten Management wird häufig zwischen defensivem und offensivem Umweltmanagement differenziert. Eine solche Dichotomie beruht auf einer statischen, zeitpunktbezogenen Betrachtung. Sie zeigt zwei Pole und die Spannbreite möglichen ökologischen Unternehmenverhaltens auf. Wie jedoch verläuft der Weg von defensivem zu offensivem Umweltmanagement? Welche Lernund Entwicklungsprozesse durchläuft die Unternehmung hierbei? Wie kann man diese systematisieren? Sowohl für die Managementpraxis als auch für die Managementlehre handelt es sich dabei um Fragen von grosser Bedeutung. In dem vorliegenden Beitrag wird näher darauf eingegangen und versucht, erste Antworten auf die oben gestellten Fragen zu geben. Umweltmanagement soll aus einer dynamischen Perspektive betrachtet und als Lern- und Entwicklungsprozess verstanden werden, der sich in der Unternehmung abspielt, der aber auch darüber hinausgeht. Um diesen Prozess zu strukturieren, sollen zwei wichtige Kriterien unterschieden werden: Integrationsgrad ökologischer Aktivitäten und Orientierung ökologischer Aktivitäten. Das erste Differenzierungskriterium betrifft die funktionsübergreifende Integration von Masșnahmen und Programmen, das zweite die Frage nach der internen oder externen Orientierung der Massnahmen. Aus der Kombination beider Kriterien ergibt sich eine Vier-Felder-Matrix, die den Weg von defensivem zu offensivem Umweltmanagement in idealtypischer Weise kennzeichnet (Abb. 1).

\section{Von isolierten Einzelmassnahmen} zu einem integrierten Ökologiekonzept

Die Reaktion auf ökologische Probleme beginnt typischerweise mit isolierten Einzelmassnahmen, die ad hoc als Reaktion auf aktuelle Problemsituationen ergriffen werden. Aufgrund von gesetzlichen Erfordernissen, Störfällen oder Medienereignissen werden in einzelnen Bereichen Massnahmen ergriffen. Sie sind häufig technischer Natur und ändern nichts an der bestehenden Struktur von Produkten und Prozessen. Sie reduzieren ökologische Probleme nachträglich, bedingen einen Zusatzaufwand und führen damit zu einer Verschlechterung der Rentabilität (Feld I).

Erst zu einem späteren Zeitpunkt reift die Erkenntnis, dass isolierte Einzelmassnahmen bei steigender Zahl zunehmend ineffizient werden oder auch, dass sich mit ökologischen Massnahmen ökonomische Vorteile erzielen lassen. Diese Phase markiert den Übergang von Feld I zu Feld II. Nun werden erste Ökoprojekte initiiert, die sich in der Schnittmenge von Ökologie und Ökonomie befinden und bereits kurzfristig zu Kosteneinsparungen führen können. Dabei ist bspw. an Verpackungsreduktionen, Energieund Wassereinsparungen sowie an die Optimierung von logistischen Prozessen zu denken. Solche unmittelbaren Erfolgserlebnisse sind wichtig. Sie wirken motivierend und stimulierend und sind als Voraussetzung für eine weitergehende Verankerung der Ökologie in die Unternehmung anzusehen. Gerade für junge Mitarbeiter besteht die Möglichkeit, sich in diesem Bereich zu engagieren. Sollen diese Ökoprojekte eine (wettbewerbs-) strategische Stosskraft erhalten und funktionsübergreifende Wirkung innerhalb der Unternehmung entfalten, dann be-

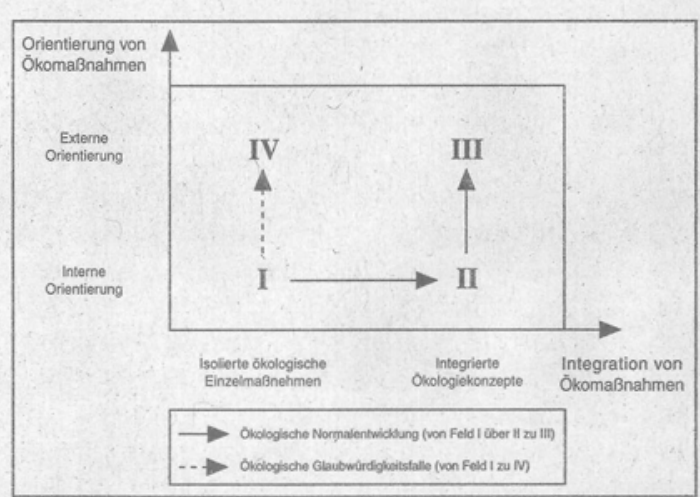

Abb. 1: Rahmen zur Erfassung ökologischer Lernund Entwicklungsprozesse von Unternehmen (Quelle: In Anlehnung an Dyllick/Belz 1994, S. 64)

darf es eines integrierenden Ökologiekonzeptes (Feld II).

In dieser Phase wird häufig ein externer Berater als »change agent « hinzugezogen und ein unternehmensweites Ökologieprojekt initiiert, an dem Mitarbeiter aus ganz unterschiedlichen $\mathrm{Be}$ reichen und Hierarchiebenen mitwirken, um einen Rahmen und eine einheitliche Ausrichtung für die verschiedenen Einzelaktivitäten zu schaffen. Mit diesem Übergang von isolierten Einzelmassnahmen zu einem integrierten Ökologiekonzept ist die Bewältigung ökologischer Herausforderungen zu einer Aufgabe geworden, die einer unmittelbaren Verantwortungsübernahme durch die oberste Führung bedarf. Dabei handelt es sich um eine notwendige, nicht aber hinreichende Voraussetzung für einen erfolgreichen ökologischen Wandel: Sollen die Lernprozesse die gesamte Unternehmung erfassen, dann bedarf es einer umfassenden Einbindung der Mitarbeiter im Sinne der (partizipatorischen) Organisationsentwicklung (1). Die Geschäftsleitung kann zwar zusammen mit externen Beratern als Initiator und Katalysator dieses Prozesses dienen, getragen werden muss er aber von jedem einzelnen Mitarbeiter. Diejenigen Mitarbeiter, die bereits in den ersten Ökopro- jekten mitgewirkt haben, können hier als ökologische Know-how-Träger und Multiplikatoren dienen. Dadurch wird individuelles ökologisches (Fach-) Wissen weitergegeben und verallgemeinert. Durch eine Integration in bestehende Strukturen und Prozesse wird Ökologie so zu einem festen und normalen Bestandteil im Unternehmenshandeln. Mit dem Übergang von Feld I zu Feld II werden ökologische Lernprozesse beschrieben, die vor allem innerhalb der Unternehmung, dem ökologischen Handlungsfeld Betrieb, stattfinden und im unmittelbaren Einflussbereich der Unternehmung liegen.

\section{Von der internen zur externen Orientierung}

Mit dem Übergang von Feld II zu Feld III sollen ökologische Lernprozesse beschrieben werden, die unternehmensübergreifend sind und sich auf das gesamte Produktleben »von der Wiege bis zur Bahre« beziehen. Damit findet eine Verlagerung von der (unternehmens-) internen zur externen Orientierung statt. Ein solcher Perspektivenwechsel hat weitreichende Folgen für die Unternehmung, die sowohl personeller als auch instrumenteller Art sind. Wurden in der ersten Phase allenfalls punktuell ökologische »change agents« aus Beratung und Wissenschaft hinzugezogen, so gilt es in der zweiten Phase auch andere externe Anspruchsgruppen in den Prozess mit einzubeziehen. Dabei ist zum einen an die Akteure entlang des ökologischen Produktlebenszyklus zu denken (Lieferanten, Absatzmittler, Kunden und Spediteure), zum anderen aber auch an andere relevante Anspruchsgruppen (wie bspw. Umweltschutz- und Konsumentenorganisationen). Erst wenn diese mit einbezogen werden, kann man die żentralen Umweltprobleme entlang des ökologischen Produktlebenszyklus identifizieren, bewerten und gemeinsam angehen.

Für die Unternehmung als wichtiges Glied in dieser Kette besteht die Aufgabe, diesen Prozess $\mathrm{zu}$ initiieren und aktiv voranzutreiben. Eine Möglichkeit ist in der Schaffung von entsprechenden Kommunikationsforen zu sehen, eine andere im Einbezug von ökologischen Kritikern in entsprechende Projekte. Hierzu ein illustratives Beispiel: Die grösste kanadische Lebensmittelkette, »Loblaw«, hat 1989 zwei Umweltschutzorganisationen hinzugezogen, um eine ökologische Produktlinie aufzubauen. Mit über 60 Mio US\$ Umsatz im ersten Jahr war die Einführung von »President's Choice G.R.E.E. N. « ein Erfolg, der ohne die Zusammenarbeit mit den ökologischen Kritikern nicht möglich gewesen wäre. Erst dadurch hat »Loblaw « die notwendige Glaubwürdigkeit für seine Produkte erlangt. Durch die Schaffung eines ökologischen Netzwerkes und die Realisierung eines aktiven ökologischen Anspruchsgruppenmanagements ist es dem kanadischen Handelsunternehmen gelungen, die Regeln des Wettbewerbs neu zu 
bestimmen und Positionierungsvorteile zu erzielen. Damit wandelt sich Ökologie von einem reinen Kosten- zu einem strategischen Wettbewerbsfaktor. Durch den Einbezug unterschiedlicher Anspruchsgruppen kann ein kollektiver Lernprozess vorangetrieben werden (»stakeholder based learning «), der nicht nur die Unternehmung, sondern auch deren Kontext transformiert. Auf instrumenteller Ebene sind in der zweiten Phase Produktlinienanalysen, Produktökobilanzen und umfassende ökologische Branchenstrukturanalysen (2) von besonderer Relevanz. Diese Instrumente sind prozessorientiert einzusetzen und dienen als Basis für weitere Diskussionen mit externen Anspruchsgruppen.

Abschliessend kann im Hinblick auf die Matrix in Abb. 1 postuliert werden, daß die ökologische Normalentwicklung einer Unternehmung von Feld I über Feld II zu Feld III verläuft. Die unternehmensinternen ökologischen Lernprozesse (Übergang von Feld I zu Feld II) sind der Ausgangspunkt und das Fundament für weiterreichende unternehmensübergreifende ökologische Lernprozesse. Wie eingangs erwähnt, handelt es sich dabei um einen idealtypischen Verlauf: In der Realität können sich unternehmensinterne und unternehmensübergreifende ökologische Lernprozesse mehr oder weniger stark überlappen. Der direkte Übergang von Feld I zu Feld IV allerdings ist als Irrweg anzusehen und wird als ökologische Glaubwürdigkeitsfalle bezeichnet. Hier werden die internen Lernprozesse umgangen und man versucht sich ohne tatsächliche Anpassungen mittels Öko-Marketing und PR zu profilieren. Eine solche Vorgehensweise kann rasch als oberflächliches »Pseudo-Ökomarketing « entlarvt werden. Bumerang-Effekte sind vorprogrammiert. Demgegenüber verlangt eine glaubwürdige Öko-Strategie, dass zunächst das »eigene Haus « betriebsökologisch in Ordnung gebracht wird, bevor man sich mittels Ökomarketing produktökologisch »aus dem Fenster lehnt «.

Thomas Dyllick, Frank Belz, St. Gallen

1) Vgl. zur Organisationsentwicklung im ökologischen Kontext Pfriem, R. (1992).2) Vgl. dazu das kürzlich erschienene Buch von Dyllick, T. et al. (1994).

\section{Literatur}

- Dyllick, T./Belz, F. 1994: Ökologische Unternehmensentwicklung: die Entstehung einer dynamischen Perspektive. In: Kreikebaum, H. u. a. (Hg.) Unternehmenserfolg durch Umweltschutz. Rahmenbedingungen, Instrumente, Praxisbeispiele. Wiesbaden 1994, S. 53-69

- Dyllick, T. et al. (1994): Ökologischer Wandel in Schweizer Branchen. Stuttgart, Bern 1994

- Pfriem, R. (1992): Ökologische Organisationsentwicklung von Unternehmen. In: Seidel, E. (Hrsg.) Betrieblicher Umweltschutz: Landschaftsökologie und Betriebswirtschaftslehre. Wiesbaden, S. $81-108$

\section{Kriterien für die Umwelt-Lernfähigkeit von Unternehmen}

Umweltorientierte Unternehmenspolitik hat in der Praxis sehr viele verschiedene Gesichter und wird sehr unterschiedlich in der Theorie beschrieben. Zum einen existieren Checklisten, wie Umweltschutz im Unternehmen umzusetzen ist, zum anderen werden Regelwerke, Normen und Richtlinien erarbeitet, die Unternehmen zur Umsetzung von Umweltschutzmaßnahmen und zum Umweltmanagement veranlassen sollen. Desweiteren erfordern ordnungspolitische Rahmenbedingungen umweltorientiertes Handeln, das meistens in Form technischer Umweltschutzmaßnahmen umgesetzt wird.

Dieses Reagieren von Unternehmen wird - je nach dem wie schnell und problemorientiert es geschieht - mit »offensivem «, »defensivem «, »aktivem «, "proaktivem « und »integriertem" Umweltmanagement beschrieben. Nur, was sagen diese Beziehungen über das Umwelt-Lernen der Organisation aus? Wenn heute vom Roll-back der Ökologie gesprochen wird und es zur Zeit der wirtschaftlichen Rezession vermehrt zu defensiven Reaktionen kommt, kann dies als ein Ausdruck von Lernfähigkeit der Unternehmen in bezug auf die Umweltproblematik gedeutet werden?

Genau wie Erfolgskriterien sind Lernkriterien subjektive Begriffe. Ein Lernen von Organisationen kann in vielerlei Reaktionstypen sichtbar werden und ist meist ein stufenartiger Prozeß. Die Reaktionsformen des Unternehmens auf den zunehmenden öffentlichen Druck werden als Lernkurve des Unternehmens beschrieben, wenn sie zu einer stufenförmigen $»$ Höherentwicklung « der Organisation führen. Dieser Lernweg kann von der passiven Opposition (z.B. Bekämpfung der Normanhebung) und Anpassung (z. B. langsame und stille Ökologisierung) bis hin zur Selbstorganisation (z.B. durch Übernahme der Initiative durch eine umweltaktive Geschäftsführung, zumindest partiell über das verbindliche Niveau hinaus) führen (vgl. Hildebrandt, 1992, S. 4). Diese Stufenleiter von Reaktionstypen muß nicht in jedem Einzelfall durchlaufen werden, sie charakterisiert nur verschiedene Lernpfade von Betrieben. Auch kann es in den einzelnen Organisationseinheiten zu unterschiedlichen Lernstufen kommen.

Im folgenden werde ich an einigen Beispielen aus einem Unternehmen verschiedene Lernprozesse beschreiben, die durch innerbetriebliche Schulungsmaßnahmen zum Umweltschutz ausgelöst wurden. Bei dem Unternehmen handelt es sich um die Schering AG, ein chemisch-pharmazeutisches Unternehmen mit ca. 7.500 Mitarbeiterinnen und Mitarbeitern in Berlin. Ich selbst war dort vier Jahre Mitarbeiterin in dem Fachbereich Zentrale Sicherheit und Umweltschutz und zuständig für Motivation und Schu- lung der Mitarbeiterinnen und Mitarbeiter im Umweltschutz.

\section{Beispiel Umweltkolloquium}

Meine Aufgabe bestand u.a. darin, eine Umwelt-Kolloquiumsreihe zu entwickeln. Ein Ziel war es, namhafte Wissenschaftler, Praktiker und Politiker als Referenten zu gewinnen, die Umweltthemen unter ihren verschiedenen $\mathrm{Ge}$ sichtspunkten darstellen sollten. Die Kolloquiumsreihe, die mit je sechs Veranstaltungen pro Jahr für drei Jahre geplant war, sollte immer von dem für Umweltschutz zuständigen Vorstandsmitglied eröffnet und von demselben Moderator begleitet werden. Dieser hatte die Aufgabe, den »roten Faden « der ausgewählten Themen, die Hauptargumente der Diskussion und den Bezug zum Unternehmen herzustellen. Die Zielgruppen, die als TeilnehmerInnen zu den Veranstaltungen eingeladen wurden, waren Führungskräfte des Unternehmens und ihre MitarbeiterInnen, die nach folgenden Kriterien ausgewählt wurden: Es sollten vor allem die Abteilungsleiter und Betriebsleiter erreicht werden, die wir auch durch die Inhalte der Vorträge zum Umwelthandeln motivieren wollten.

Ausdrücklich wurden keine externen Personen eingeladen, damit die anschließenden Diskussionen sich ohne Einschränkung auf interne Umsetzungen beschränken konnten. Damit diese Veranstaltung nicht eine "nur angenehme" Vortragsveranstaltung war, sondern es tatsächlich auch zu einem Lernen der Organisation kommen konnte, wurde für jede Veranstaltung ein sogenannter »Pate « aus dem Unternehmen bestimmt. Dieser Pate hatte jeweils die Aufgabe, ein Pate der Gedanken, der Diskussion und Forderungen zu sein. D.h., er hatte nach den Veranstaltungen die Aufgabe, zu erkunden, zu fragen, zu analysieren, ob die erhobenen Forderungen, Ideen, Fragestellungen etc. für das eigene Unternehmen eine Herausforderung darstellen.

Um diese Ziele zu überprüfen, organisierte ich am Ende der ersten Kolloquiumsreihe eine Veranstaltung mit dem Vorstand, den Paten, dem Leiter des Fachbereichs Sicherheit und Umweltschutz und dem Moderator. Wir wollten die Lern- und Erfahrungsergebnisse nach einem Jahr Umweltkolloquium zusammenfassen und Ziele für das nächste Jahr diskutieren. Inzwischen läuft das Kolloquium im vierten Jahr und wird in diesem Jahr in dieser Form beendet werden. Alle Veranstaltungen einschließlich der Diskussion wurden veröffentlicht, und die Broschüren sind für alle Mitarbeiter erhältlich. Dieses Beispiel zeigt meiner Meinung nach einen Lernprozeß, der auf verschiedenen Ebenen initiert wurde und der letztlich zur Selbstorganisation im oben erwähnten Sinne führen kann. 
(c) 20I0 Authors; licensee IÖW and oekom verlag. This is an article distributed under the terms of the Creative Commons Attribution Non-Commercial No Derivates License (http://creativecommons.org/licenses/by-nc-nd/3.o/), which permits unrestricted use, distribution, and reproduction in any medium, provided the original work is properly cited. 